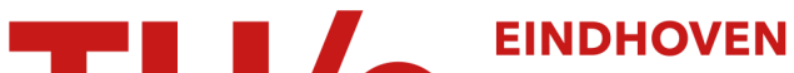 \\ UNIVERSITY OF \\ TECHNOLOGY
}

\section{Cooperative adaptive cruise control, design and experiments}

\section{Citation for published version (APA):}

Naus, G. J. L., Vugts, R. P. A., Ploeg, J., Molengraft, van de, M. J. G., \& Steinbuch, M. (2010). Cooperative adaptive cruise control, design and experiments. In Proceedings of the 2010 American Control Conference (ACC 2010), 30 June 30 - 2 July 2010, Baltimore, Maryland, USA (pp. 6145-6150)

https://doi.org/10.1109/ACC.2010.5531596

DOI:

10.1109/ACC. 2010.5531596

Document status and date:

Published: 01/01/2010

\section{Document Version:}

Accepted manuscript including changes made at the peer-review stage

\section{Please check the document version of this publication:}

- A submitted manuscript is the version of the article upon submission and before peer-review. There can be important differences between the submitted version and the official published version of record. People interested in the research are advised to contact the author for the final version of the publication, or visit the $\mathrm{DOI}$ to the publisher's website.

- The final author version and the galley proof are versions of the publication after peer review.

- The final published version features the final layout of the paper including the volume, issue and page numbers.

Link to publication

\section{General rights}

Copyright and moral rights for the publications made accessible in the public portal are retained by the authors and/or other copyright owners and it is a condition of accessing publications that users recognise and abide by the legal requirements associated with these rights.

- Users may download and print one copy of any publication from the public portal for the purpose of private study or research.

- You may not further distribute the material or use it for any profit-making activity or commercial gain

- You may freely distribute the URL identifying the publication in the public portal.

If the publication is distributed under the terms of Article 25fa of the Dutch Copyright Act, indicated by the "Taverne" license above, please follow below link for the End User Agreement:

www.tue.nl/taverne

Take down policy

If you believe that this document breaches copyright please contact us at:

openaccess@tue.nl

providing details and we will investigate your claim. 


\title{
Cooperative adaptive cruise control, design and experiments
}

\author{
Gerrit Naus, René Vugts, Jeroen Ploeg, René van de Molengraft, Maarten Steinbuch
}

\begin{abstract}
The design of a CACC system and corresponding experiments are presented. The design targets string stable system behavior, which is assessed using a frequency-domain-based approach. Following this approach, it is shown that the available wireless information enables small inter-vehicle distances, while maintaining string stable behavior. The theoretical results are validated by experiments with two CACC-equipped vehicles. Measurement results showing string stable as well as string unstable behavior are discussed.
\end{abstract}

\section{INTRODUCTION}

Cooperative Adaptive Cruise Control (CACC) is an extension of Adaptive Cruise Control (ACC) functionality. ACC automatically adapts the velocity of a vehicle if there is preceding traffic. Commonly, a radar or lidar is used to detect preceding traffic, enabling automatic following of a predecessor. As ACC is primarily intended as a comfort system, and, to a smaller degree, as a safety system, a relatively large inter-vehicle distance has to be adopted [2], [3]. Decreasing this distance to a small, preferably constant value of only several meters is expected to yield an increase in traffic throughput, and, specifically focusing on heavy duty vehicles, a significant reduction of the drag force, thus decreasing fuel consumption [1]. To enable this for a string of vehicles while maintaining so-called string stability, standard ACC functionality has to be extended with intervehicle communication [9]. Extending ACC functionality with wireless inter-vehicle communication is called Cooperative ACC (CACC). In Fig. 1, a schematic representation of a string of vehicles, a so-called platoon, equipped with CACC functionality is shown.

This research focuses on the design of a CACC system using communication with the directly preceding vehicle only, as opposed to communication with multiple preceding vehicles or with a designated platoon leader. This is often called semi-autonomous ACC and facilitates easy implementation, see Fig. 1 [9]. Furthermore, heterogeneous traffic is considered, i.e., vehicles with different characteristics [4], [10], and delay in the communicated signal is taken into account [6], [7], [11]. Finally, the communication will be implemented as a feedforward signal. Hence, if no communication is present, standard ACC functionality is still available [9], [12].

G.J.L. Naus, R.P.A. Vugts, M.J.G. v.d. Molengraft and M. Steinbuch are with the Department of Mechanical Engineering, Control Systems Technology group, Technische Universiteit Eindhoven, P.O. box 513, Eindhoven, The Netherlands $\{g . j .1$.naus, r.p.a.vugts, m.j.g.v.d.molengraft, m.steinbuch\}@tue.nl. J. Ploeg is with the Business Unit Automotive, Department of Integrated Safety, TNO Science and Industry, P.O. box 756, Helmond, The Netherlands jeroen.ploegetno.nl.

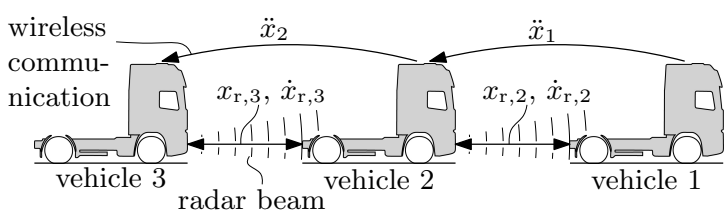

Fig. 1. Schematic representation of a platoon of vehicles equipped with CACC functionality, where $x_{r, i}, \dot{x}_{r, i}$ and $\ddot{x}_{i}$ represent the relative position, the relative velocity and the acceleration of vehicle $i$, respectively.

The design and analysis of CACC systems generally evolve around the notion of string stability. The string stability of a platoon indicates whether oscillations are amplified upstream, i.e., from the leading vehicle $i=1$ to vehicle $i>1$ in the platoon. String stability is extensively discussed in literature. As a result, various definitions are present, see, e.g., [4], [6], [7], [9], [10], [14]. The main ambiguity concerns the signals to consider. Either the control input, the vehicle output or state, the error, or a combination of these is considered. In previous work [8], it is concluded that the vehicle output or state has to be considered when heterogeneous traffic is taken into account in the analysis.

Following the new approach presented in [8], a frequencydomain-based framework for the design of a CACC system and the definition of string stability is presented in Sect. II. Using this framework as an analysis tool, in Sect. III the influence of the available wireless information as well as the choice for the inter-vehicle spacing policy are discussed, focusing on string stability of the system.

The main contribution of this paper involves the experimental validation of the presented framework. In Sect. IV, the implementation of the CACC system on a real vehicle platform is presented, according to the concept depicted in Fig. 1. The measurement results validate the assumptions made in the modeling of the system and the results of the theoretical string stability analysis. The paper is closed with conclusions and an outlook on future work.

\section{PROBLEM FORMULATION}

\section{A. Control structure}

Consider a string of vehicles, see Fig. 1. The primary control objective for each vehicle is to follow the corresponding preceding vehicle at a desired distance $x_{r, d, i}(t)$

$$
x_{r, d, i}(t)=r_{i}+h_{d, i} \dot{x}_{i}(t), \quad \text { for } i \geq 1
$$

where $r_{i}$ is the desired distance at standstill, $h_{d, i}$ is the socalled desired headway time, and $\dot{x}_{i}(t)$ is the measured velocity of vehicle $i$. To anticipate high-frequent measurement 
noise, the velocity signal is filtered using a first-order lowpass filter $f(\cdot)$ with cut-off frequency $\omega_{f, i}$, yielding

$$
x_{r, d, i}(t)=r_{i}+h_{d, i} f\left(\dot{x}_{i}(t)\right), \quad \text { for } i \geq 1
$$

The headway time is the time it takes for vehicle $i$ to reach the current position of the preceding vehicle $i-1$ when continuing to drive with a constant velocity. The radar output data, $x_{r, i}(t)$ and $\dot{x}_{r, i}(t)$, is used in a feedback setting by a standard ACC controller. The acceleration of the preceding vehicle $\ddot{x}_{i-1}(t)$ is available via wireless communication, and is used in a feedforward setting. The first vehicle in the string, $i=1$, is assumed to follow a given timevarying reference position $x_{0}(t)$. The resulting control setup is depicted schematically in Fig. 2.

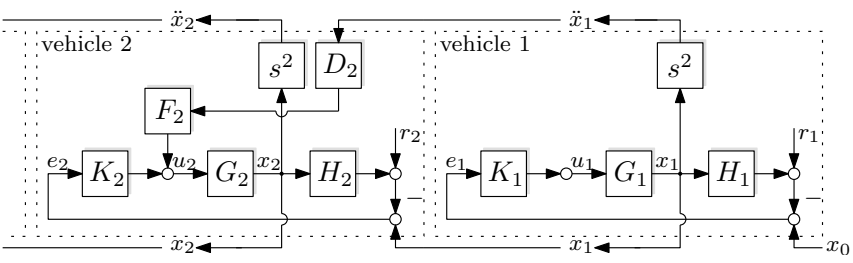

Fig. 2. Control structure of a platoon, where $G_{i}$ the dynamics of the $i^{t h}$ vehicle, $K_{i}$ the corresponding ACC feedback controller, $F_{i}$ the feedforward controller, $D_{i}$ the communication delay and $H_{i}$ the spacing policy dynamics, for $i=1,2, \ldots$.

Consider the setup depicted in Fig. 2. The model $G_{i}(s)$ represents a closed-loop system, including a controller $K_{l, i}$ for the longitudinal vehicle dynamics. Hence, the input $u_{i}(t)$ of $G_{i}(s)$ can be regarded as a desired acceleration. The controller $K_{l, i}$ ensures tracking of this desired acceleration via actuation of the throttle and brake system. In a generic form, the corresponding closed-loop dynamics of each vehicle are represented as

$$
G_{i}(s)=\frac{k_{G, i}}{s^{2}\left(\tau_{i} s+1\right)} e^{-\phi_{i} s}, \quad \text { for } i \geq 1
$$

where $\tau_{i}^{-1}=\omega_{G, i}$ is the corresponding closed-loop bandwidth, $\phi_{i}$ is the actuator delay time, and $k_{G, i}$ is the loop gain, which equals 1 for an appropriately designed feedback controller $K_{l, i}$. The spacing error between the desired distance $x_{r, d, i}(t)$ and the actual distance $x_{r, i}(t)$ equals $e_{i}(t)=$ $x_{r, i}(t)-x_{r, d, i}(t)$, where $x_{r, i}(t)=x_{i-1}(t)-x_{i}(t)$, and $x_{r, d, i}(t)$ as defined in (2). Defining $e_{i}(t)$ in this manner implies that positive control action, i.e., acceleration, is required when the inter-vehicle distance $x_{r, i}(t)$ is too large with respect to the desired distance $x_{r, d, i}(t)$, which is intuitive. Taking, for simplicity, $r_{i}=r=0$, the corresponding Laplace transform, denoted by $\mathcal{L}(\cdot)$, equals

$$
\mathcal{L}\left(e_{i}(t)\right)=E_{i}(s)=X_{i-1}(s)-H_{i}(s) X_{i}(s), \quad \text { for } i \geq 1
$$

where

$$
H_{i}(s)=1+h_{d, i} \frac{\omega_{f, i}}{s+\omega_{f, i}} s, \quad \text { for } i \geq 1
$$

represents the so-called spacing policy dynamics. For $r_{i} \neq 0$, the subsequent analysis does not change.
Given the vehicle dynamics $G_{i}(s)$ (3) and the spacing policy dynamics $H_{i}(s)$ (5), a feedback controller with PD action provides the freedom to choose the closed-loop bandwidth of the closed-loop system $T_{i}(s)$ (from $x_{i-1}-r_{i}$ to $H_{i} x_{i}$ )

$$
T_{i}=\frac{H_{i} G_{i} K_{i}}{1+H_{i} G_{i} K_{i}}
$$

Correspondingly, the ACC feedback controller $K_{i}(s)$ is defined as

$$
K_{i}(s)=\omega_{K, i}\left(\omega_{K, i}+s\right), \quad \text { for } i \geq 1
$$

where $\omega_{K, i}$ the breakpoint of the controller.

The wireless communication includes delay, which is represented by a constant delay time $\theta_{i}$, yielding $\mathcal{L}\left(\ddot{x}_{i-1}\left(t-\theta_{i}\right)\right)=D_{i}(s) s^{2} X_{i-1}(s)$, where

$$
D_{i}(s)=e^{-\theta_{i} s}, \quad \text { for } i>1
$$

The acceleration of the preceding vehicle is used as a feedforward control signal via a feedforward filter $F_{i}(s)$. The design of this feedforward filter is based on inverse model dynamics. From (6), it follows directly that the uncontrolled open-loop transfer equals $L_{i}(s)=H_{i}(s) G_{i}(s) D_{i}(s) s^{2}$. It is assumed that an estimator for the delayed acceleration signal is not present. Consequently, demanding $\mathcal{L}\left(e_{i}(t)\right)=0$, the feedforward filter is given by

$$
F_{i}=\left(H_{i} G_{i} s^{2}\right)^{-1}, \quad \text { for } i>1
$$

\section{B. String stability, a frequency-domain approach}

The design and analysis of CACC systems generally evolve around the notion of string stability. Different definitions of string stability are present in literature, see, e.g., [4], [6], [7], [9], [10], [14]. To facilitate a decentralized controller design, focusing on feasibility of implementation, a definition that is independent of other traffic is desirable. Correspondingly, when string stability of a platoon of heterogeneous traffic is considered, amplification of oscillations in the vehicle state, i.e., the position, velocity or acceleration, have to be considered [8]. Accordingly, the transfer function from the input $\mathcal{L}\left(x_{0}(t)\right)=X_{0}(s)$ to $X_{i}(s)$ is given by

$$
\frac{X_{i}}{X_{0}}= \begin{cases}G_{1} K_{1} S_{1}, & \text { for } i=1 \\ \frac{X_{1}}{X_{0}} \prod_{k=2}^{i} S_{k}\left(F_{k} D_{k} s^{2}+K_{k}\right) G_{k}, & \text { for } i>1\end{cases}
$$

where

$$
S_{i}=\left(1+H_{i} G_{i} K_{i}\right)^{-1}
$$

is the closed-loop sensitivity corresponding to vehicle $i$. These relations follow directly from the block diagram in Fig. 2. Focus is on the magnitude of the so-called stringstability transfer function $\hat{S} S_{i}(s)$

$$
\hat{S S}{ }_{i}=\frac{X_{i}}{X_{1}}=\frac{X_{i}}{X_{0}}\left(\frac{X_{1}}{X_{0}}\right)^{-1}, \quad \text { for } i>1
$$

where $i=1$ is not considered, as vehicle 1 does not receive wireless information of the preceding reference vehicle. The magnitude of $\hat{S S}{ }_{i}$ is a measure for the amplification of 
$x_{0}(t)$ upstream the platoon. A necessary condition for string stability thus is

$$
\left|\hat{S S_{i}}(j \omega)\right| \leq 1, \quad \text { for } i>1, \forall \omega
$$

To fulfill condition (13) for vehicle $i>2$, the dynamics of all vehicles $k \in\{1, \ldots, i-1\}$ have to be known. Considering heterogeneous traffic, this requires an extensive communication structure. As communication with the directly preceding vehicle only is considered, a more conservative condition for string stability is defined as

$$
\left|S S_{i}(j \omega)\right| \leq 1, \quad \text { for } i>1, \forall \omega
$$

where

$$
S S_{i}=\frac{X_{i}}{X_{i-1}}, \quad \text { for } i>1
$$

As it holds that

$$
\hat{S S_{i}}=\prod_{k=2}^{i} S S_{k}, \quad \text { for } i>1
$$

condition (13) is automatically satisfied if (14) is satisfied. Hence, (14) is a sufficient condition for string stability. Condition (13) considers the platoon as a whole: if compensated somewhere else, local string-unstable behavior can be allowed in the platoon. Condition (14), on the other hand, imposes string stable behavior at every position in the platoon. Combining (10) and (15) yields the string stability transfer function $S S_{i}(s)$

$$
S S_{i}=\frac{G_{i} F_{i} D_{i} s^{2}+G_{i} K_{i}}{1+H_{i} G_{i} K_{i}}, \quad \text { for } i>1
$$

A sufficient condition for string stability of a platoon of heterogeneous vehicles is thus defined by (14). Comparing this to the string stability definitions in [9], [11]-[14], amplification of oscillations in the vehicle state is considered, rather than oscillations in the distance error. As a result, the definition of string stability (14) targets preventing so-called shockwave behavior, rather than preventing collisions.

\section{SYSTEM ANALYSIS FOCUSING ON STRING STABILITY}

Consider the CACC system setup as presented in Sect. IIA. The design variables are the feedback controller $K_{i}(s)$, the feedforward filter $F_{i}(s)$ and the spacing policy dynamics $H_{i}(s)$. Focusing on string stability (14), in this section, the influence of the design of $H_{i}(s)$ and $F_{i}(s)$ is evaluated. It is assumed that the feedback controller $K_{i}(s)$ as defined in (7) is used. Furthermore, considering an appropriate controller $K_{l, i}$ and assuming $\phi_{i}=0$, ideal vehicle dynamics result, yielding $G_{i}(s)=s^{-2}$. The consequences of this assumption with respect to practice will be evaluated later on.

\section{A. Constant inter-vehicle spacing}

To start with, consider the case of no feedforward, i.e., $F_{i}(s)=0$. Constant inter-vehicle spacing implies $h_{d, i}=0$, yielding $H_{i}(s)=1$. Without feedforward, an ACC system instead of a CACC system results. The output string stability transfer function (17) reduces to

$$
S S_{i}=\frac{G_{i} K_{i}}{1+G_{i} K_{i}}=T_{i}^{*}, \quad \text { for } i>1
$$

where $T_{i}^{*}(s)$ is the complementary sensitivity of the resulting system. Considering $T_{i}^{*}(s)$, in practice, sensor noise or model errors will impose high-frequent rolloff, resulting in an increased magnitude at other frequencies. Correspondingly, a peak will be present in the complementary sensitivity $T_{i}^{*}(s)$, due to the well-known Bode-sensitivity-integral constraint. Hence, a peak will be present in the string stability transfer function as well. Consequently, in case of an ACC system, string stability can not be guaranteed for a constant intervehicle spacing.

In the case that the feedforward filter is taken into account, a CACC system results, where $F_{i}(s)=1$ for $H_{i}(s)=1$ and $G_{i}(s)=s^{-2}$ (see (9)). For the sake of clarity, communication delay is not taken into account, i.e., $D_{i}(s)=1$. The output string stability transfer function (17) then equals

$$
S S_{i}=\frac{1+G_{i} K_{i}}{1+G_{i} K_{i}}=1, \quad \text { for } i>1
$$

Hence, only marginal string stability $\left|S S_{i}(j \omega)\right|=1$, for $i>$ $1, \forall \omega$, can be achieved. Marginal in this case indicates that the design is not robust for uncertainties or modeling errors. For example, taking into account time delay as well would mean that no string stability can be achieved.

\section{B. Velocity-dependent inter-vehicle spacing, ACC case}

Consider the spacing-policy dynamics $H_{i}(s)$ as defined in (5). In this case, a velocity-dependent inter-vehicle spacing is adopted. In the case of no feedforward, i.e., $F_{i}(s)=0$, in which an ACC system instead of a CACC system results, this yields

$$
S S_{i}=\frac{G_{i} K_{i}}{1+H_{i} G_{i} K_{i}}, \quad \text { for } i>1
$$

Considering real-valued frequencies $\omega \in \mathbb{R}$ only, substitution of $G_{i}(s)=s^{-2}, K_{i}(s)$ (7) and $H_{i}(s)$ (5) in (20) shows that string stability can be guaranteed if

$$
h_{d, i}^{2}+2 \omega_{K, i}^{-1} h_{d, i}-2 \omega_{K, i}^{-2} \geq \frac{\omega^{2}}{\omega_{K, i}^{4}}, \quad \text { for } i>1, \forall \omega
$$

where $\omega_{f, i}=\omega_{K, i}$. This particular choice for $\omega_{f, i}$ actually improves the string stability characteristics of the system, which is not discussed further at this point. Considering realvalued frequencies $\omega \in \mathbb{R}$ implies $\min \left\{\omega^{2}\right\}=0$. Hence, finding a minimum headway time $h_{d, i, \min }$ for $h_{d, i}$ in (21), yields

$$
h_{d, i}^{2}+2 \omega_{K, i}^{-1} h_{d, i}-2 \omega_{K, i}^{-2} \geq 0, \quad \text { for } i>1
$$

From (22) it follows directly that string stability can be guaranteed for $\omega_{K, i} h_{d, i} \geq 3^{1 / 2}-1 \approx 0.73$, for $i>1$.

Suppose that $\omega_{K, i}=0.5 \mathrm{rad} \mathrm{s}^{-1}$, then $h_{d, i} \geq h_{d, i, \min } \approx$ $1.46 \mathrm{~s}$ has to hold to ensure string stability. This is illustrated in Fig. 3. In this figure, simulation results are shown corresponding to a platoon of three vehicles following a 
reference vehicle. For $h_{d, i}=2.0 \mathrm{~s}$ (the upper figure), all vehicles in the platoon follow the reference vehicle while actually decreasing the amplitude of the velocity signal. For $h_{d, i}=0.5 \mathrm{~s}$ (the lower figure), however, the amplitude of the velocity signal is indeed amplified upstream the platoon, yielding string unstable behavior.

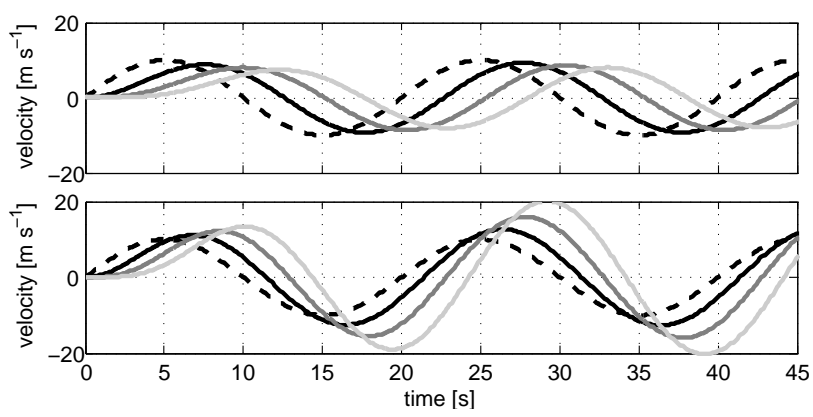

Fig. 3. Simulation results of a platoon of three ACC-equipped vehicles following a reference vehicle, represented by the dashed line. The solid lines represent the vehicles $i=1$ to $i=3$ for the dark to the light colored lines, respectively. The results in the upper and lower figure correspond to $h_{d, i}=2.0 \mathrm{~s}$ and $h_{d, i}=0.5 \mathrm{~s}$, for $i \in\{1,2,3\}$, respectively.

\section{Velocity-dependent inter-vehicle spacing, CACC case}

For the CACC case, where $F_{i}(s)$ is as defined in (9), the string stability transfer function (17) equals

$$
S S_{i}=\frac{D_{i}+H_{i} G_{i} K_{i}}{H_{i}\left(1+H_{i} G_{i} K_{i}\right)}, \quad \text { for } i>1
$$

Not taking into account communication delay, i.e., $D_{i}=1$, yields

$$
S S_{i}=\frac{1}{H_{i}}, \quad \text { for } i>1
$$

Consequently, string stability can be guaranteed for any $h_{d, i}>0$. For $h_{d, i}=0$, only marginal string stability can be achieved (see Sect. III-A). In Fig. 4, the simulation results of a platoon of vehicles where $h_{d, i}=0.5 \mathrm{~s}$ is used, are shown. As these results show, all vehicles in the platoon follow the reference vehicle while decreasing the amplitude of the velocity of preceding vehicles in the platoon, as opposed to the corresponding results shown in Fig. 3 in which an ACC system is employed.

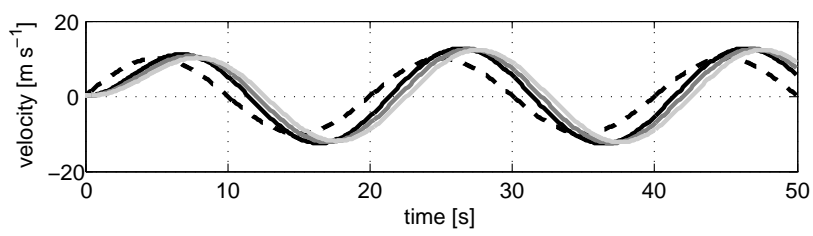

Fig. 4. Simulation results of a platoon of three CACC-equipped vehicles following a reference vehicle, represented by the dashed line. The solid lines represent the vehicles $i=1$ to $i=3$ for the dark to the light colored lines, respectively. The results correspond to $h_{d, i}=0.5 \mathrm{~s}$, for $i \in\{1,2,3\}$.

Taking into account communication delay as well, i.e., $D_{i}(s)$ as defined in (8), a minimum value $h_{d, i, \min }$ for $h_{d, i}$ is required to guarantee string stability. Depending mainly on $\theta_{i}$, the minimum required headway time $h_{d, i, \min }$ for which string stability can still be guaranteed, is significantly smaller in the case of CACC than in the case of ACC; as (24) indicates, $\lim _{\theta_{i} \rightarrow 0} h_{d, i, \min }\left(\theta_{i}\right)=0$. Further analysis of $h_{d, i, \min }\left(\theta_{i}\right)$ is not discussed here.

\section{EXPERIMENTAL VALIDATION}

To validate the theory, experiments are performed using two vehicles. For budget reasons, equipment of more vehicles was not possible. The use of only two vehicles instead of a larger platoon requires extrapolation of the results. However, if the theory is validated for the first two vehicles in a platoon, additional vehicles with the same CACC system will show the same behavior. Hence, the theory can be validated using only two vehicles. The models for the communication delay $D_{i}(s)$ and the vehicle models $G_{i}(s)$ are identified using measurements. Based on these models, the design of $K_{i}(s), F_{i}(s)$ and $H_{i}(s)$ is discussed. Finally, three different experiments are executed.

\section{A. Experimental setup}

Two Citroën C4's are used as testing platform, see Fig. 5. For the wireless inter-vehicle communication, the standard Wi-Fi protocol IEEE $802.11 \mathrm{~g}$ is used, with an update rate of $10 \mathrm{~Hz}$. The acceleration of vehicle 1 is derived from the ESP system and communicated to vehicle 2 . The communication delay equals on average $10 \mathrm{~ms}$. Using a zero-order hold approach for the communicated signal, combination of the corresponding delay and the communication delay yields $\theta_{i} \approx 60 \mathrm{~ms}$ as a total delay for $D_{i}(s)$ (8). GPS time stamping is adopted to synchronize the measurements of the two vehicles.

Vehicle 2 is equipped with a customized brake-by-wire system and a corresponding controller for the longitudinal dynamics of the vehicle [5]. Furthermore, an OMRON laser radar with $150 \mathrm{~m}$ range is built-in. The CACC system is implemented at $100 \mathrm{~Hz}$ on a dSpace AutoBox using rapid control prototyping.

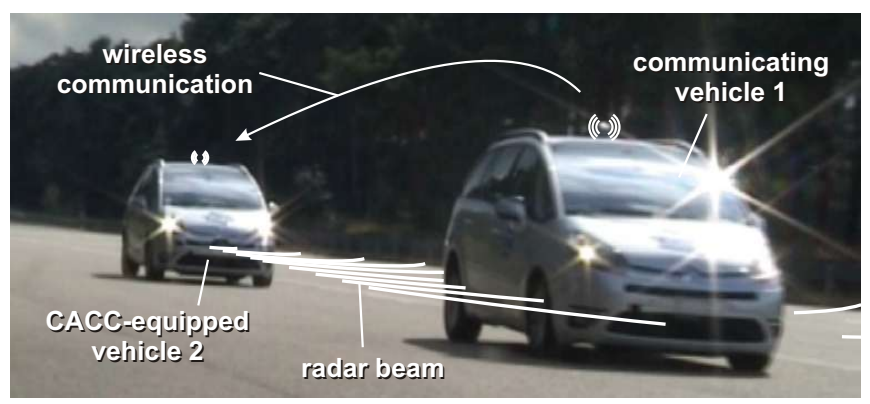

Fig. 5. Experimental setup.

\section{B. Vehicle model identification}

In the problem setup presented in Sect. II, it is assumed that the internal closed-loop dynamics of the vehicle has bandwidth $\omega_{G, i}$. In practice, however, the controller $K_{l, i}$ for 
the longitudinal vehicle dynamics consists of a feedforward part only. Hence, in this case, the generic model for $G_{i}(s)$ (3) represents a feedforward controller incorporating mass compensation, a low-pass filter with cut-off frequency $\omega_{G, i}$, and actuator delay $\phi_{i}$. The main difference with respect to a feedback controller is the low-frequent gain $k_{G, i} \neq 1$.

The gain $k_{G, i}$, the time constant $\tau_{i}=\omega_{G, i}^{-1}$, and the delay time $\phi_{i}$ are identified using step response measurements, see Fig. 6. The simulation results in Fig. 6 correspond to the model $G_{i}^{*}(s)=G_{i}(s) s^{2}$ where $k_{G, i}=0.9, \tau_{i}=$ $0.2(\mathrm{rad} / \mathrm{s})^{-1}$ and $\theta_{i}=0.2 \mathrm{~s}$. The main characteristics of the closed-loop vehicle model are covered appropriately. Hence, the model $G_{i}(s)$ is a sufficient model to describe the vehicle dynamics including the controller for the longitudinal vehicle dynamics.
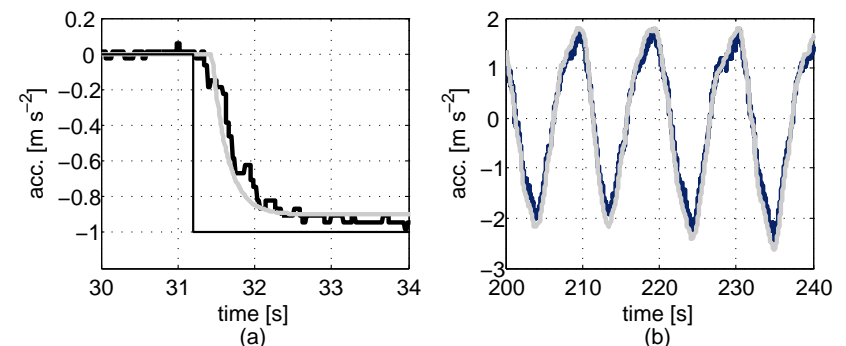

Fig. 6. (a) identification step response data, and (b) validation data. Measurement results (black), corresponding simulation results (grey) with the model $G_{i}^{*}(s)=G_{i}(s) s^{2}$, and the step input signal (thin black).

\section{CACC design}

The implemented feedback controller $K_{i}(s)$ is a combination of the PD-controller (7) and a first-order low-pass filter to prevent amplification of high-frequent noise. The breakpoint of the PD-controller lies at $\omega_{K, i}=0.5 \mathrm{rad} \mathrm{s}^{-1}$. The cut-off frequency of the low-pass filter equals half the sample frequency, i.e., $100 \pi \mathrm{rad} \mathrm{s}^{-1}$. Hence, the influence on the closed-loop system is negligible in the frequency range of interest. Analogous to the analysis in Sect. III-B, the cutoff frequency of the low-pass filter $f(\cdot)$ (see (2)) equals $\omega_{f, i}=\omega_{K, i}$.

For the design of the feedforward controller $F_{i}(s)$ (9), ideal vehicle dynamics are assumed, i.e., $G_{i}(s)=s^{-2}$, yielding $F_{i}(s)=H_{i}^{-1}(s)$. This corresponds to the analysis presented in Sect. III. The identified vehicle model, however, is not ideal. In Fig. 7, Bode magnitude plots of the corresponding string stability transfer function $S S_{i}(s)$ are shown, for $h_{d, i}=0.5 \mathrm{~s}$. Considering ideal vehicle dynamics, $F_{i}(s)=H_{i}^{-1}(s)$ would yield string stable dynamics. However, considering the identified model, the resulting dynamics are string unstable.

\section{String stability experiments}

To validate the theory of Sect. III, three experiments are performed, see Table I. In Fig. 8, the Bode magnitude plots corresponding to the setups used in Experiments $i$ and ii are shown. In both experiments, wireless information is not taken

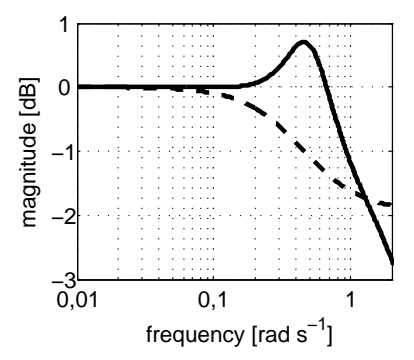

Fig. 7. Bode magnitude plots of $\left|S S_{i}(j \omega)\right|$, considering an ideal vehicle model (dashed black), and the identified model (solid black).

TABLE I

OVERVIEW OF THE EXPERIMENTS.

\begin{tabular}{|c|c|c|}
\hline Experiment & communication & $h_{d, i}[\mathrm{~s}]$ \\
\hline i & no & 2.0 \\
ii & no & 0.5 \\
iii & yes & 0.5 \\
\hline
\end{tabular}

into account. Consequently, no feedforward filter is present and an ACC system results. Based on the theory (see Sect. III-B), the system should exhibit string stable behavior in Experiment $\mathrm{i}$, as $h_{d, i}=2.0>\omega_{K, i}^{-1}(\sqrt{3}-1) \approx 1.46 \mathrm{~s}$, whereas string unstable behavior is expected in Experiment ii, as $h_{d, i}=0.5<1.46 \mathrm{~s}$. The Bode magnitude plots of $S S_{i}(s)$ show corresponding results; $\left|S S_{i}(j \omega)\right| \leq 0$ holds for Experiment i, which is not the case for Experiment ii.

In Fig. 9 and 10, measurement results for Experiments $\mathrm{i}$ and ii are shown. For Experiment i, the amplitude of the oscillations in the velocity of vehicle 2 is smaller than that of vehicle 1 , which corresponds to the anticipated string stable behavior. Analogously, for Experiment ii, the behavior of vehicle 2 is string unstable; the oscillations in the velocity of vehicle 1 are amplified by vehicle 2 . However, the corresponding acceleration of vehicle 2 shows saturated behavior at $2 \mathrm{~m} \mathrm{~s}^{-2}$ (Fig. 10). Based on the linear, frequencydomain-based analysis, no theoretical guarantees regarding string stability of the corresponding behavior can be given, although, in practice, the concept still seems to hold.

In Fig. 11, measurement results for Experiment iii are shown. The wireless information is used to determine an additional feedforward control signal. Hence, a true CACC

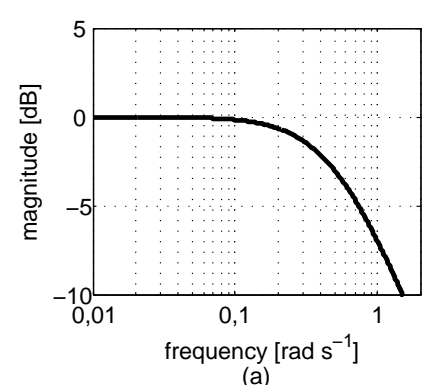

(a)

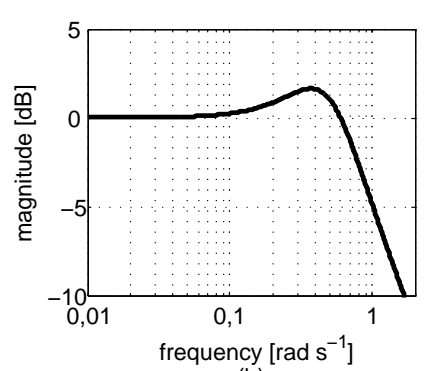

(b)
Fig. 8. Bode magnitude plots of $\left|S S_{i}(j \omega)\right|$, for (a) Experiment i, and (b) Experiment ii. 


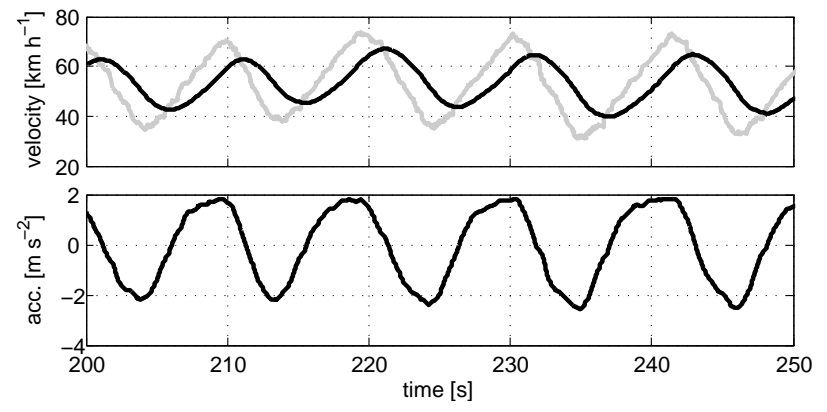

Fig. 9. Measurement results of Experiment i. In the upper figure, the velocity of vehicle 1 (grey) and vehicle 2 (black) are shown. In the lower part of the figure, the acceleration of vehicle 2 is shown.

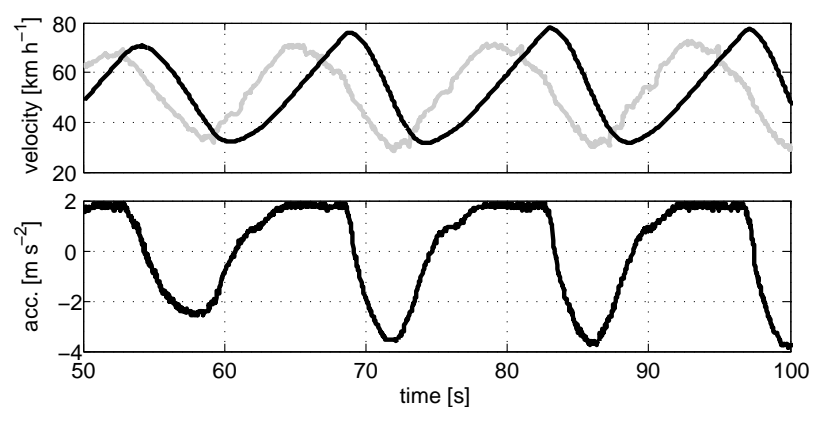

Fig. 10. Measurement results of Experiment ii. In the upper figure, the velocity of vehicle 1 (grey) and vehicle 2 (black) are shown. In the lower part of the figure, the acceleration of vehicle 2 is shown.

system is tested. Based on the theory, the system should exhibit string unstable behavior, see Fig. 7. The measurement results in Fig. 11, however, show that vehicle 2 does not amplify the oscillations in the velocity, indicating string stable behavior. Moreover, as the corresponding acceleration signal, again, shows saturated behavior, no theoretical guarantees can be given regarding string stability of the system. Nevertheless, the effectiveness of the proposed CACC setup is evident. Comparing the results of the ACC system in Experiment ii and the CACC system in Experiment iii clearly illustrates the potential of the proposed design.

\section{CONCLUSiOnS AND FUTURE WORK}

A theoretical framework for the frequency-domain analysis and design of a CACC system is presented. The framework enables assessment of the string stability characteristics of the system. Theoretical analysis of the CACC system shows that a velocity-dependent spacing policy is required to achieve string stable system behavior. Furthermore, it is shown that the feedforward controller enables small intervehicle distances, while maintaining string stability.

Implementation of the CACC system on a real vehicle platform is presented. Measurement results validate the assumptions made in the modeling and the theoretical results of the string stability analysis. However, limitations of the implementation of the CACC system on a practical setup are also shown, such as the presence of saturation and non-

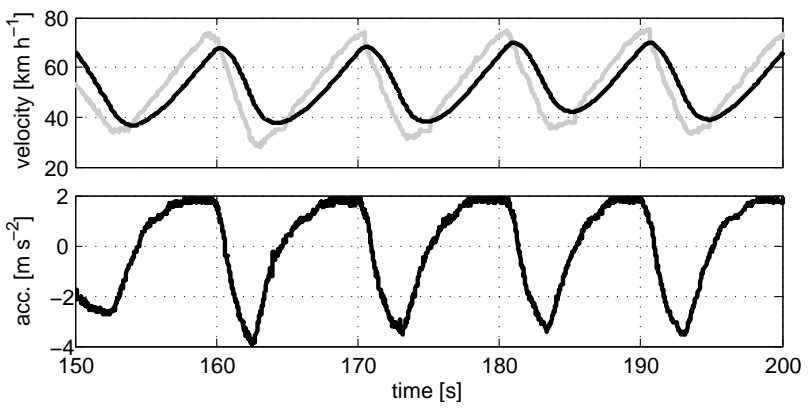

Fig. 11. Measurement results of Experiment iii. In the upper figure, the velocity of vehicle 1 (grey) and vehicle 2 (black) are shown. In the lower part of the figure, the acceleration of vehicle 2 is shown.

ideal low-level vehicle dynamics. Using the proposed, linear approach, theoretical guarantees regarding string stability can only be given for linear vehicle models.

Further experimental validation of the concept is part of future research. Furthermore, research focuses on taking into account saturations as well as including robustness against model uncertainties.

\section{REFERENCES}

[1] B. Van Arem, C.J.G. Van Driel, and R. Visser. The impact of Cooperative Adaptive Cruise Control on traffic flow characteristics. IEEE Trans. Intell. Transp. Syst., 7(4):429-436, December 2006.

[2] International Organization for Standardization. Transport information and control systems - Adaptive Cruise Control systems - Performance requirements and test procedures. ISO 15622, October 2002.

[3] P.A. Ioannou and C.C. Chien. Autonomous intelligent cruise control. IEEE Trans. Veh. Technol., 42(4):657-672, November 1993.

[4] M.E. Khatir and E.J. Davison. Decentralized control of a large platoon of vehicles using non-identical controllers. In Proc. 2004 Am. Control Conf., pages 2769-2776, Boston, Massachusetts, July 2004.

[5] D.O. De Kok. Adaptive cruise control with stop\&go: documentation of the vehicle controller. TNO report, March 2004. TNO Automotive, Helmond, The Netherlands.

[6] X. Liu and S.S. Mahal. Effects of communication delay on string stability in vehicle platoons. In Proc. IEEE Intell. Transp. Syst. Conf., pages 625-630, August 2001.

[7] X.Y. Lu, S. Shladover, and J.K. Hedrick. Heavy-duty truck control: Short inter-vehicle distance following. In Proc. Am. Control Conf., volume 5, pages 4722-4727, Boston, Massachusetts, July 2004.

[8] G.J.L. Naus, R.P.A. Vugts, J. Ploeg, M.J.G. Van de Molengraft, and M. Steinbuch. Towards on-the-road implementation of cooperative adaptive cruise control. In Proc. 16th World Congr. and Exhib. Intell. Transp. Syst. Serv., Stockholm, Sweden, September 2009.

[9] R. Rajamani and C. Zhu. Semi-autonomous Adaptive Cruise Control systems. IEEE Trans. Veh. Technol., 51(5):1186-1192, Sep. 2002.

[10] E. Shaw and J.K. Hedrick. Controller design for string stable heterogeneous vehicle strings. In Proc. 46th IEEE Conf. Decis. Control, pages 2868-2875, December 2007.

[11] S. Sheikholeslam and C.A. Desoer. A system level study of the longitudinal control of a platoon of vehicles. J. Dyn. Sys., Meas., Control, 114(2):286-292, June 1992.

[12] D. Swaroop and J.K. Hedrick. Constant spacing strategies for platooning in automated highway systems. J. Dyn. Sys., Meas., Control, 121:462-470, 1999.

[13] D. Swaroop, J.K. Hedrick, C.C. Chien, and P.A. Ioannou. A comparison of spacing and headway control strategy for automatically controlled vehicles. Veh. Syst. Dyn., 23(8):597425, November 1994.

[14] D. Swaroop, J.K. Hedrick, and S.B. Choi. Direct adaptive longitudinal control of vehicle platoons. IEEE Trans. Veh. Technol., 50(1):150-161, January 2001. 